\title{
ChIP goes dynamic
}

Chromatin interaction dynamics are quantified using a modified ChIP method that measures cross-linking kinetics.

Chromatin immunoprecipitation, or ChIP, is a widely used method that has yielded countless insights into gene regulation by determining where chromatinbinding proteins latch onto DNA sequences. Formaldehyde is used to cross-link these interactions in cells; the chromatin is isolated and fragmented; and an antibody specific to a chromatin-binding protein of interest is used to pull down the DNA sequences, which are then identified by PCR, microarray or sequencing.

For all its power, however, ChIP provides little information about the stability or dynamics of protein-DNA interactions. "I was impressed by the quantity and quality of data that was accumulating that told us where factors bind to chromatin, but we were still somehow left wanting in terms of our understanding of how to interpret it, or take that and translate it into a deeper understanding of mechanism," says David Auble of the University of Virginia Health System. Live-cell imaging studies have shown that many chromatin interactions are short lived, but imaging methods cannot pinpoint binding locations with high resolution.

With the goal of making more biochemically rigorous ChIP measurements in cells, Auble, a biologist, teamed up with his colleague Stefan Bekiranov, a physicist and bioinformatics expert. The result, after nearly 5 years of work, is an approach that mathematically describes the dependence of the ChIP signal on formaldehyde cross-linking time, a method the researchers call crosslinking kinetic analysis, or CLK, pronounced like "clock." The overall on-rate, off-rate and fraction of bound chromatin at steady state can be extracted in such an experiment, enabling the half-life of a chromatin complex of interest to be calculated.

Working in yeast, Auble's team used a quench-flow apparatus to analyze formaldehyde-treated samples with subsecond time resolution. After establishing that CLK was working as intended with a series of crucial control experiments, the researchers analyzed Gal4 binding to the GAL3 promoter in a proof-of-principle test. They found that the half-life of the Gal4-GAL3 interaction was about 10 minutes; but the promoter occupancy was low, which suggested that a single complex triggers multiple rounds of transcription initiation and that the GAL3 gene is transcribed in infrequent bursts. They also analyzed two transcription factors whose dynamic behaviors were independently validated with fluorescence recovery after photobleaching experiments.

The researchers then looked at the dynamic interactions of the TATA-binding protein (TBP) with seven different promoters driven by RNA polymerases I, II or III. They were particularly interested in studying the effect of mutations to Mot1, an enzyme that dissociates the TBP-DNA interaction. They found that, as expected from biochemical evidence, Mot1 mutations increased the TBP ChIP signal at RNA polymerase II promoters. However, further experiments showed that these mutant interactions were unstable, and CLK analysis showed that TBP binding was far more dynamic in Mot1 mutant cells. Overall, this indicated that the role of Mot1 is to dissociate weakly bound TBP complexes in order to facilitate more stable binding. "It completely turned our understanding of the phenomenon on its head," says Auble. "The enzyme is doing the same thing we always thought it did-it's just not targeting the complexes we thought it was targeting: it's targeting these weakly bound, transcriptionally inactive ones."

Auble plans to continue to improve the CLK approach, both by refining the kinetic model to make it more accurate and by streamlining the experimental approach to make it less labor intensive and to potentially scale up to genome-wide analyses. $\mathrm{He}$ is hopeful that others will adopt the method to address other interesting sorts of questions, such as whether transcription complexes are dynamic at one type of promoter and how features of dynamics correlate with transcription output and chromatin structure. "To me, it would be very satisfying if people felt that it was something generally useful and were even able to improve upon it and modify it for whatever it is they were interested in doing," he says.

\section{Allison Doerr}

\section{RESEARCH PAPERS}

Poorey, K. et al. Measuring chromatin interaction dynamics on the second time scale at single-copy genes. Science 342, 369-372 (2013). 